\title{
Analysis of normal-appearing white matter of multiple sclerosis by tensor-based two-compartment model of water diffusion
}

\author{
Yasuhiko Tachibana • Takayuki Obata - Mariko Yoshida • Masaaki Hori • Koji Kamagata • \\ Michimasa Suzuki • Issei Fukunaga • Kouhei Kamiya • Kazumasa Yokoyama • \\ Nobutaka Hattori • Tomio Inoue • Shigeki Aoki
}

Received: 28 October 2014 /Revised: 2 December 2014 / Accepted: 15 December 2014 / Published online: 11 January 2015

(C) The Author(s) 2015. This article is published with open access at Springerlink.com

\begin{abstract}
Objectives To compare the significance of the twocompartment model, considering diffusional anisotropy with conventional diffusion analyzing methods regarding the detection of occult changes in normal-appearing white matter (NAWM) of multiple sclerosis (MS).

Methods Diffusion-weighted images (nine b-values with six directions) were acquired from 12 healthy female volunteers (22-52 years old, median 33 years) and 13 female MS patients (24-48 years old, median 37 years). Diffusion parameters based on the two-compartment model of water diffusion considering diffusional anisotropy was calculated by a proposed method. Other parameters including diffusion tensor imaging and conventional apparent diffusion coefficient (ADC) were also obtained. They were compared statistically between the control and MS groups.

Results Diffusion of the slow diffusion compartment in the radial direction of neuron fibers was elevated in MS patients $\left(0.121 \times 10^{-3} \mathrm{~mm} 2 / \mathrm{s}\right)$ in comparison to control $(0.100 \times$
\end{abstract}

\footnotetext{
Y. Tachibana $\cdot$ T. Obata $(\bowtie)$

Research Center for Charged Particle Therapy, National Institute of Radiological Sciences, 4-9-1 Anagawa, Inage-ku, Chiba 263-8555, Japan

e-mail: t_obata@nirs.go.jp

Y. Tachibana $\cdot \mathrm{T}$. Inoue

Department of Radiology, Yokohama City University Graduate

School of Medicine, 3-9 Fukuura, Kanazawa-ku,

Yokohama 236-0004, Japan

Y. Tachibana $\cdot$ M. Yoshida $\cdot$ M. Hori $\cdot$ K. Kamagata $\cdot$ M. Suzuki $\cdot$

I. Fukunaga $\cdot$ K. Kamiya $\cdot$ S. Aoki

Department of Radiology, Juntendo University School of Medicine,

2-1-1 Hongo, Bunkyo-ku, Tokyo 113-8431, Japan

K. Yokoyama $\cdot$ N. Hattori

Department of Neurology, Juntendo University School of Medicine,

2-1-1 Hongo, Bunkyo-ku, Tokyo 113-8431, Japan
}

$\left.10^{-3} \mathrm{~mm}^{2} / \mathrm{s}\right)$, the difference being significant $(P=0.001)$. The difference between the groups was not significant in other comparisons, including conventional ADC and fractional anisotropy (FA) of diffusion tensor imaging.

Conclusion The proposed method was applicable to clinically acceptable small data. The parameters obtained by this method improved the detectability of occult changes in NAWM compared to the conventional methods.

Key Points

- Water diffusion was compared between the controls and multiple sclerosis patients.

- A two-compartment model, considering diffusional anisotropy was selected for water diffusion analysis.

- Axial and radial diffusion of fast and slow diffusion components were evaluated.

- A new method was developed to obtain the metrics stably.

- The metrics indicated high detectability of slight differences between the groups.

Keywords Multiple sclerosis - Diffusion tensor imaging · Brain · Magnetic resonance imaging · Computer-assisted image processing

$\begin{array}{ll}\text { Abbreviations } \\ \text { MS } & \text { Multiple sclerosis } \\ \text { ADC } & \text { Apparent diffusion coefficient } \\ \text { NAWM } & \text { Normal-appearing white matter } \\ \text { Df } & \begin{array}{l}\text { Diffusion coefficient of the fast diffusion } \\ \text { compartment }\end{array} \\ & \text { Diffusion coefficient of the slow diffusion } \\ \text { Ds } & \text { compartment } \\ \text { fs } & \text { Fraction of the slow diffusion compartment } \\ \text { DTI } & \text { Diffusion tensor imaging } \\ \text { MPG } & \text { Motion probing gradient } \\ \text { DWI } & \text { Diffusion-weighted imaging } \\ \text { EPI } & \text { Echo planar imaging }\end{array}$




$\begin{array}{ll}\text { ROI } & \text { Region of interest } \\ \text { CSF } & \text { Cerebrospinal fluid } \\ \text { FA } & \text { Fractional anisotropy of diffusion } \\ \text { eDWI } & \text { Estimated diffusion-weighted image }\end{array}$

\section{Introduction}

In multiple sclerosis (MS), diffusion analysis of water molecules using magnetic resonance imaging (MRI) is a wellknown quantitative technique for revealing specific microstructural changes [1-3]. It enables the apparent diffusion coefficient (ADC) in the tissues to be calculated by the following mono-exponential equation using two different bvalues:

$S_{b} / S_{0}=\exp (-b D)$,

where $\mathrm{S}_{\mathrm{b}}$ and $\mathrm{S}_{0}$ indicate the signals with and without motionprobing gradients (MPG), $b$ indicates the b-value, and D indicates ADC [4]. Its high sensitivity to occult tissue damage in normal-appearing white matter (NAWM) of MS has been well discussed [5-7].

On the other hand, it is also well known that signal changes do not exactly follow the above equation (Eq. 1) in vivo. Thus, another model that considers two separate diffusion components (fast and slow components) has been well discussed. This two-compartment model is given by this bi-exponential equation:

$s_{b} / s_{0}=\left(1-f_{s}\right)^{*} \exp \left(-b D_{f}\right)+f s^{*} \exp \left(-b D_{s}\right)$,

where Df and Ds indicate the diffusion coefficients of the fast and slow diffusion components, respectively, and fs indicates the fraction of the slow diffusion compartment [4]. This equation (Eq. 2) is known to fit the experimental signal changes better than Eq. 1, and thus is often adopted for discussing in vivo water diffusion [4, 8-12]. Of note, this equation is also well known as an intra-voxel incoherent motion (IVIM) model, which usually implements the division of water diffusion from water perfusion in low-b-value data. On the other hand, the two-compartment model used in this study divides the fast and slow diffusion components from the data acquired in low to very high b-values. In this respect, the models are different.

Diffusion tensor imaging (DTI) is a widely used technique that provides anisotropy of the diffusion of water molecules which furnishes the indices of ADC in three dimensions. A previous study found that DTI provides additional information to the usual ADC to quantify the extent and pathological severity of the structural changes occurring within MS plaques and in NAWM [13].

The idea and the technique of DTI may also be applied to Df, Ds, and fs of the two-compartment model (Eq. 2) separately to add information of diffusion anisotropy to them. It will enable us to observe the parameters separately in axial and radial directions, which may provide detailed information of the changes in cell structure and character. It is discussed that water permeability and diffusion restriction of cell membrane is reflected in these parameters [4]. In this regard, it should be better observed in the specific radial direction that is perpendicular to the membrane. It may be useful for detailed diagnosis of MS, because those membrane characteristics may change during the accumulation of diffuse chronic inflammation and demyelination of this disease [1]. However, the technique has not been well discussed. One reason might be that the post-processing operation becomes rather complicated for obtaining adequate results without great noise and errors if the technique is applied straightforward to the small data acquired in clinics. In addition, the complicated postprocessing is time-consuming, which also makes it a problem for daily use. In this study, we proposed a new data processing procedure to compensate for these problems. The purpose of this study is to compare the significance of the twocompartment model considering diffusional anisotropy obtained by this proposed method with conventional diffusion analysis regarding the detection of occult changes in NAWM of MS patients.

Table 1 Major parameters of diffusion-weighted imaging sequence

\begin{tabular}{|c|c|}
\hline MRI system & 3 T Achieva; Philips Medical Systems \\
\hline Coil & 8-channel-array SENSE head coil \\
\hline b-values ${ }^{\mathrm{a}}$ & $\begin{array}{l}0,124,496,1116,1983,3099,4463,6074 \\
\quad 7934,10,041,12,397,15,000 \mathrm{~s} / \mathrm{mm}^{2}\end{array}$ \\
\hline $\begin{array}{l}\text { Diffusion encoding } \\
\text { directions }\end{array}$ & 6 \\
\hline$\delta / \Delta$ & $37.8 / 47.3 \mathrm{~ms}$ \\
\hline Acquisition & Single-shot, spin-echo planar imaging \\
\hline $\begin{array}{l}\text { Repetition time /Echo } \\
\text { time }\end{array}$ & $4000 / 96 \mathrm{~ms}$ \\
\hline Number of signals & one \\
\hline Section thickness & $4 \mathrm{~mm}$ \\
\hline Number of Slices & 10 slices \\
\hline Field of view & $256 \times 256 \mathrm{~mm}$ \\
\hline Matrix ${ }^{\mathrm{b}}$ & $64 \times 64$ \\
\hline Scan time & $5 \mathrm{~min}$ \\
\hline
\end{tabular}

${ }^{\text {a }}$ Data from an applied b-value over $8000 \mathrm{~s} / \mathrm{mm}^{2}$ were excluded from this study

${ }^{\mathrm{b}}$ Matrix was low to avoid low signal-to-noise-ratio in higher b-values $\delta$ : Duration time of the motion probing gradient; $\Delta$, interval time between the motion probing gradients 


\section{Materials and methods}

This retrospective clinical study was approved by our local ethics committee. It was based on a search of our local clinical database: all the data assessed in this study (diffusionweighted imaging, DWI) were previously acquired as a part of daily routine for another study (unpublished), and we reanalyzed the data. Written informed consent was obtained from all patients and volunteers at that time.
Subjects

We selected 13 female MS patients (age range 2448 years, median 37 years) who had relapsing-remitting MS defined according to standard criteria [14]. Those who had either distinguishable brain atrophy or MS plaque at centrum semiovale were not included. The disease duration ranged from 1.8 to 18.4 years, median 6.0 years. In addition, 12 healthy female volunteers (age
Fig. 1 Procedure for generating axial eDWI. The schema illustrates the procedures to calculate estimated axial diffusion-weighted images (axial eDWI) and shows the virtual bvalue-dependent signal change regarding axial diffusion. a The source images are the series of DWI that were acquired with multiple b-values including zero, and multiple motion probing gradient (MPG). Each b-value except zero requires no less than six MPG encoding directions (six in this study). $\mathbf{b}$ Diffusion tensor and its eigenvalues $\left(\lambda_{1}, \lambda_{2}\right.$, and $\left.\lambda_{3}\right)$ are calculated for every $b$-pair of $b=0$ and each b-value. $\mathbf{c}$ Estimate of axial DWI (eDWI). Virtual signal intensity $\left(\mathrm{S}_{\mathrm{k}}\right)$ at a certain b-value $(\mathrm{b}=\mathrm{k})$ could be obtained as illustrated by a mono-exponential equation with variables of signal intensity at $b=0\left(\mathrm{~S}_{0}\right)$, b-value $(\mathrm{k})$, and first eigenvalue $\left(\lambda_{1}\right)$ of the diffusion tensor calculated by the $b$-value pair of $b=0$ and $b=k$. This process needs to be repeated for every b-value to estimate the whole b-value-dependent signal change
A)

(one image for $b=0$ )
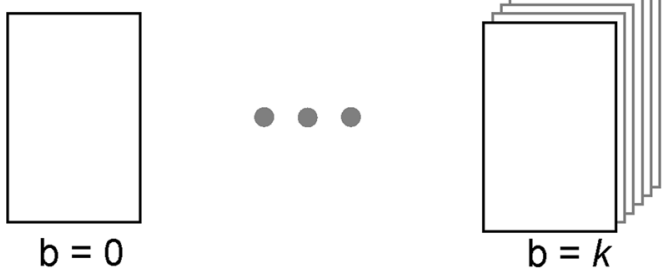

6 MPG directions

Multiple b-values

B)

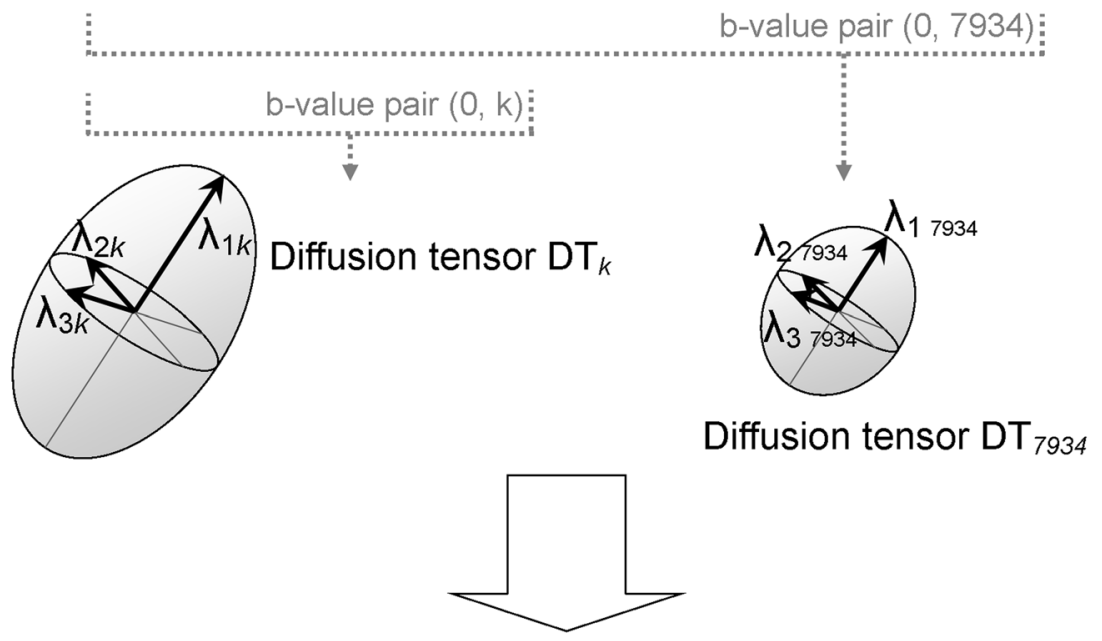

C) b-value dependent signal change regarding axial diffusion

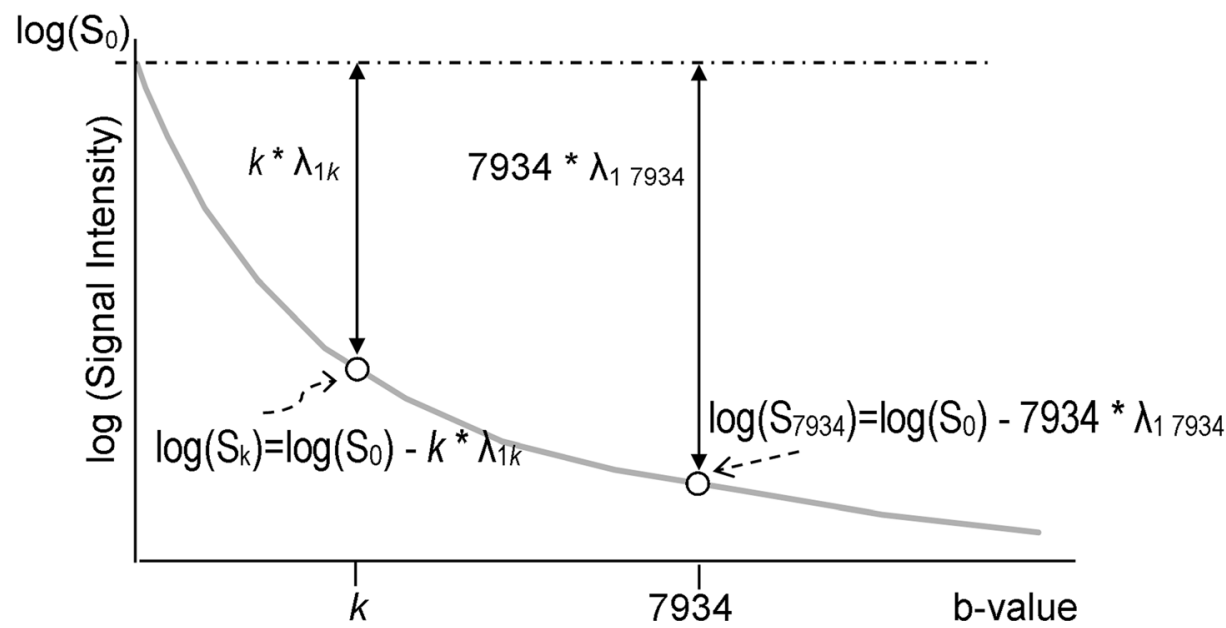


range 22-52 years, median 33 years) were added to the study as controls.

Imaging procedure and diffusion parameters

Our diffusion-weighted imaging (DWI) data were acquired on a 3 T system (Achieva; Philips Medical Systems, Best, the Netherlands). Twelve different b-values (0, 124, 496, 1116, 1983, 3099, 4463, 6074, 7934, 10,041, 12,397, 15,000 s/ $\mathrm{mm}^{2}$ ), with diffusion encoding in six different directions were applied. Other major imaging parameters are summarized in Table 1. Data from an applied b-value over $8000 \mathrm{~s} / \mathrm{mm}^{2}$ were excluded from this study to avoid the effect of image distortion and also because of concern about their low signal-to-noise ratio. We applied two semi-automated masking procedures to the data in order to select solely white matter pixels as much as possible for further assessment. First, to exclude cerebrospinal fluid (CSF) pixels, we excluded the pixels with mean ADC calculated from data of $\mathrm{b}=0$ and $1116 \mathrm{~s} / \mathrm{mm}^{2}$ of over $1.5 \times$ $10^{-3} \mathrm{~mm}^{2} / \mathrm{s}$. Second, to exclude grey matter pixels, we excluded the pixels with fractional anisotropy of diffusion (FA) [13] calculated from the same data of under 0.15 . The finally included pixels were regarded as NAWM of MS, because patients who had either distinguishable brain atrophy or MS plaque in FLAIR images were not included. After these operations, smoothing of the remaining image was done by moving average filter (eight connected neighbourhoods).

The diffusion tensor was computed from the data set of $b=$ 0 and $1116 \mathrm{~s} / \mathrm{mm}^{2}$. Axial ADC and radial ADC were obtained, which were the first eigenvalue of the diffusion tensor, and the

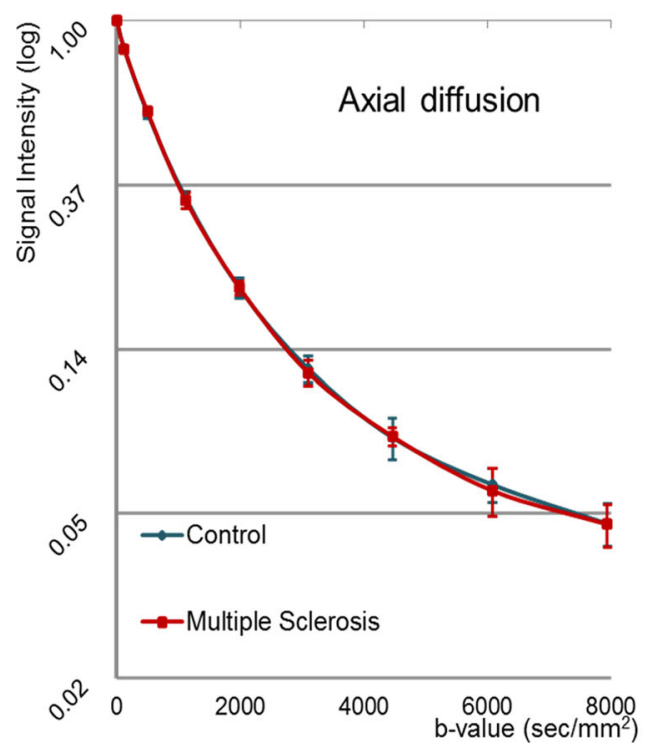

Fig. 2 b-value-dependent signal changes in axial and radial eDWI. The semi log graphs indicate the virtual b-value-dependent signal change (estimated diffusion weighted image: eDWI) regarding axial and radial diffusion, respectively. Data points and error bars indicate the averages average of the second and third eigenvalues of the tensor. Mean ADC was also obtained by applying the monoexponential equation to mean DWI (image averaged between the six images of different MPG directions) at the same bvalues. Each of the parameters of the two-compartment model in axial or radial direction (axial and radial Ds, Df, and fs) was also estimated following the steps described in the next section (Estimated DWI). In addition, mean Ds, Df, and fs were calculated by applying bi-exponential fitting (Introduction, Eq. 2) to mean DWI ( $b=0$ to $\left.7934 \mathrm{~s} / \mathrm{mm}^{2}\right)$. All the computing operations in this study were performed using our in-house software developed with a commercial analysis package (MATLAB version R2007b, Math Works Inc., Natick, MA, USA).

Estimated DWI (eDWI) and diffusion tensor-based two-compartment model

The estimated DWI (eDWI) method was designed to estimate the virtual b-value-dependent signal change regarding the proper axial or radial diffusion in each pixel. To simulate this virtual imaging, the following steps were carried out with the raw image data set (DWI with nine b-values, 0 to $7994 \mathrm{~s} / \mathrm{mm}^{2}$, in six MPG directions).

The diffusion tensor $\left(\mathrm{DT}_{\mathrm{b}}\right)$ and its eigenvalues $\left(\lambda_{1 \mathrm{~b}}, \lambda_{2 \mathrm{~b}}\right.$, and $\lambda_{3 b}$ ) were calculated pixel-by-pixel for every b-value pair that includes $b=0$ (e.g., the pair of $b=0$ and 124 , the pair of $b=$ 0 and $496 \ldots$ and the pair of $b=0$ and 7934) (Fig. 1a, b). The first (largest) eigenvalue of each tensor indicates the supposed axial diffusion coefficient of the source pixel at the applied b-

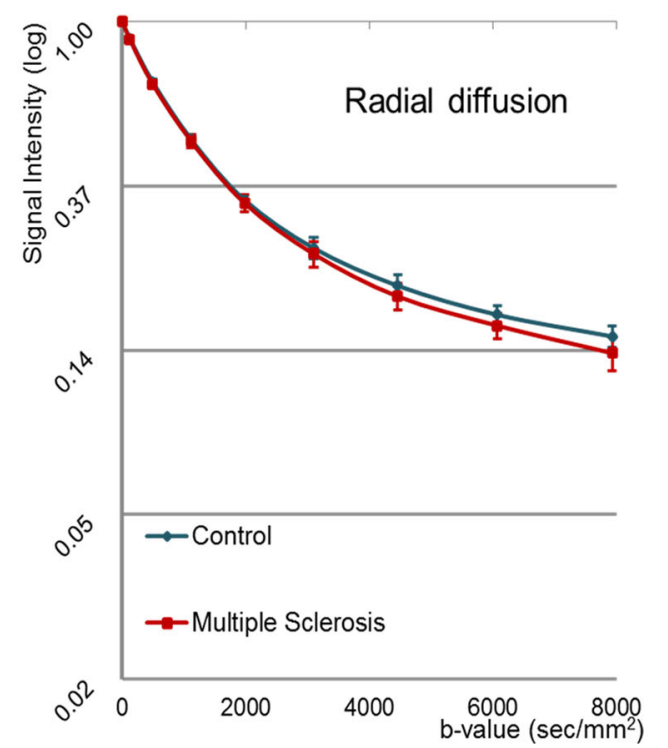

and standard deviations of the group. The b-value-dependent signal changes are consistent between the groups in axial diffusion, but in radial diffusion there was a difference in higher b-values, where the signal change of the MS group was greater than that in the control group 
value pair, so the first eigenvalue $\left(\lambda_{1 \mathrm{~b}}\right)$ was substituted for $D$ in Eq. 1 (Introduction) to obtain the virtual signal intensity $\left(S_{b}\right)$ at that b-value regarding the axial diffusion (Fig. 1c). The whole image series of eDWI regarding axial diffusion was generated by repeating the above steps for all the b-values. Equally, the virtual signal intensity regarding the radial diffusion could be generated by substituting the average of the second and third eigenvalues $\left(\lambda_{2 b}\right.$ and $\left.\lambda_{3 b}\right)$ instead of $\lambda_{1 b}$.

The two-compartment model metrics considering diffusion anisotropy, which were Ds, Df, and fs in proper axial and radial directions, were calculated by fitting bi-exponential curve (Introduction, Eq. 2) to these axial and radial eDWIs.

\section{Comparison between control and MS patients}

Two image slices at the level of the centrum semiovale were selected for comparison. The slices were defined as the adjacent two slices right above the bilateral lateral ventricles.

For comparison, first the axial and radial eDWI signal intensities were averaged in each case separately to demonstrate the $b$-value-dependent signal changes of those virtual images. Second, all the calculated diffusion parameters were averaged in each case for statistical comparison. The control and MS patient groups were compared using the MannWhitney rank sum test. $P$-value under $0.0125(=0.05 / 4)$ was considered significant to avoid type 1 errors in the multiplicity of statistical analysis.

Map images of the proposed diffusion parameters were also made.

\section{Results}

The $b$-value-dependent signal changes of axial eDWI were consistent between the groups, whereas those of radial eDWI differed between the groups in the high b-values (Fig. 2). This result matched the statistical results in which the median of radial Ds was higher in the MS group $\left(0.121 \times 10^{-3} \mathrm{~mm}^{2} / \mathrm{s}\right)$ compared to the control group $\left(0.100 \times 10^{-3} \mathrm{~mm}^{2} / \mathrm{s}\right)$ with significant difference $(P=$ $0.001)$, while there was no significant difference either in axial Ds or in Df and fs in both axial and radial directions (Table 2). The mapped images were inhomogeneous, but the results were also consistent with the statistical results (Table 2), where radial Ds was higher in the MS group compared to control, but the other metric maps were not different between the groups (Fig. 3).

The differences were not statistically significant between the groups in all conventional DWI and DTI metrics, which were axial, radial, and mean $\mathrm{ADC}$, and FA (Table 2). 

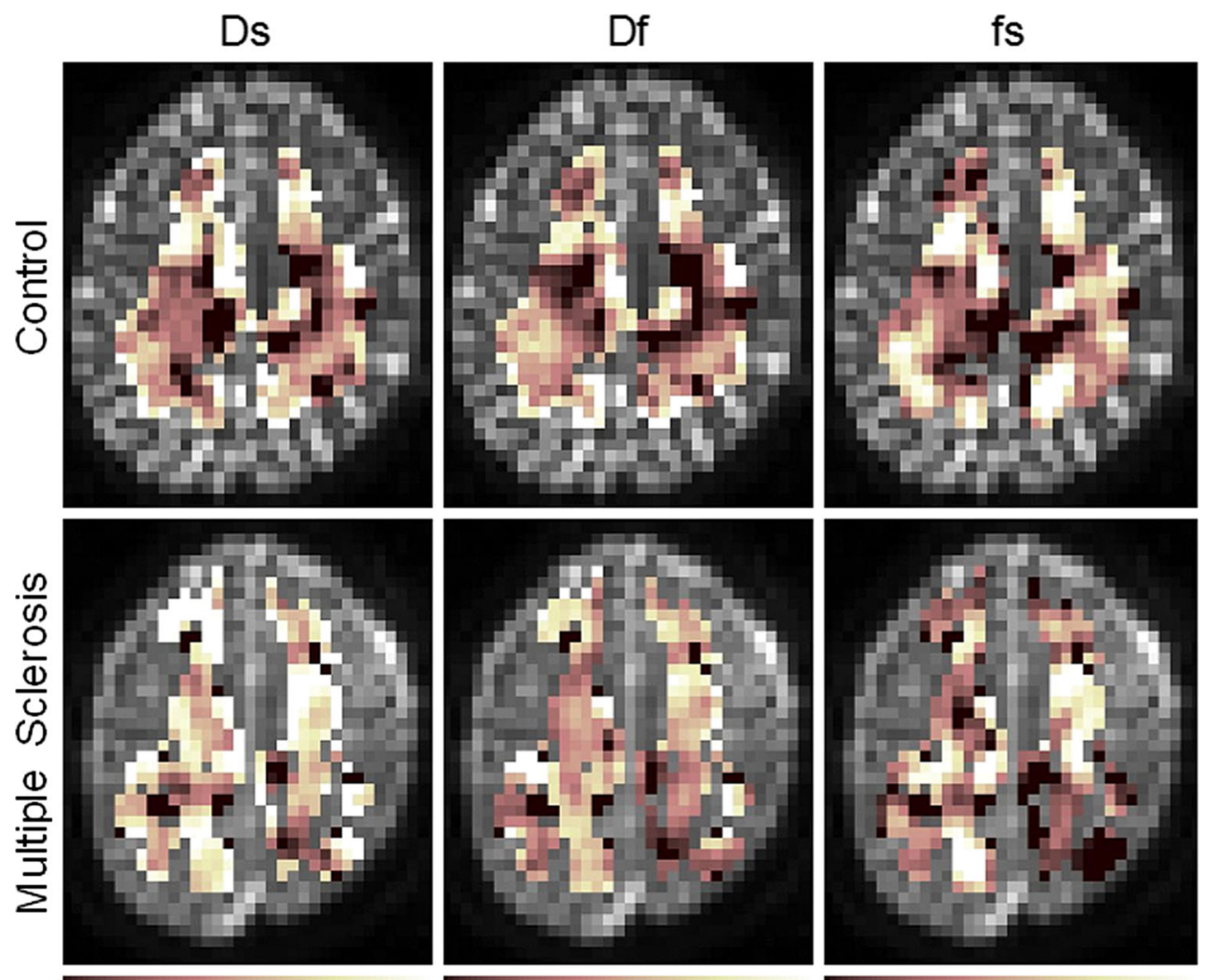

0.0498

0.1390 .850

Fig. 3 Sample images of mapped radial Ds, Df, and fs. The sample maps indicate the diffusion parameters of diffusion tensor-based two-compartment model regarding the radial diffusion: Ds (diffusion coefficient of slow diffusion component), Df (diffusion coefficient of fast diffusion component), and fs (fraction of slow diffusion component). The maps (coloured area) are superimposed on $\mathrm{a} b=0$ DWI image. The maps are restricted to the white matter area, as the cerebral spinal fluid pixels and

\section{Discussion}

The proposed method in this study, which was a combination of the "estimated DWI" based on DTI and bi-exponential curve fitting, was designed as a simplified post-processing method to obtain axial and radial Ds, Df, and fs. The previously reported conventional method $[10,12]$ requires many steps for this process: bi-exponential curve fitting for each MPG direction separately; obtaining the diffusion tensors of Ds, Df, and fs separately from its results; calculating conventional DTI to define axial and radial directions; and finally, generating each metrics by projecting each tensor to the axial and radial directions. On the other hand, the proposed method requires only the diffusion tensor at each $b$-value paired with $\mathrm{b}=0$ and two bi-exponential curve fittings (axial and radial). The bi-exponential fitting procedure is usually the most timeconsuming and error-generating procedure, so the merit of the proposed method may increase if the number of MPG encoding directions increases.

The estimated b-value-dependent signal change (eDWI) in the proposed method depends on the premise that the direction grey matter pixels were semi-automatically excluded in the masking process (see section of Materials and methods: Diffusion parameters). Colors indicate the values of the scale shown in the colour bar below. Radial Ds was higher in the MS group compared to control, but the other metric maps were not different between the groups. Of note, the parametric images were obtained at $64 \times 64$ pixels, but the pixels outside the brain parenchyma were trimmed

of the largest eigenvalue of the diffusion tensor is identical to the axial direction in all the b-value pairs used (e.g., pairs of $\mathrm{b}=0$ and each $\mathrm{b}$-value). Previous studies have reported that the major eigenvectors of the fast and slow diffusions tend to be strongly co-aligned with the known fibre tracts in the regions of high anisotropy $[10,12]$. This may support our method, as we selected the ROI by including only pixels with FA of more than 0.15. In the comparison between the control and MS groups, the b-value-dependent signal changes of eDWI were consistent in the axial direction, while a difference was seen in the high b-value areas in the radial direction (Fig. 2). These findings were confirmed by statistical comparison, in which radial Ds was higher in MS with a profoundly significant difference, but the differences in other comparisons were not significant (Table 2); Ds is the diffusion coefficient of the slower diffusion that is reflected most in the signal change at high b-values. Neither of conventional DWI and DTI metrics (axial, radial, and mean ADC by mono-exponential fitting, and FA) showed significant differences between the groups. Therefore, our suggested method may be more sensitive to the occult changes of MS. Furthermore, none of mean Ds, Df, or 
fs, which were the two-compartment model parameters without diffusion anisotropy information, showed significant differences. Thus, we may consider that the combination of diffusion anisotropy and two-compartment model was important in detecting the changes. The mean value of radial Ds is approximately $50 \%$ less than that of axial Ds, so minimal changes in radial Ds might have been concealed by the larger axial Ds when they were not observed separately.

On the other hand, the map image of Ds, Df, and fs in the control group was still inhomogeneous (Fig. 3). One plausible reason is that there were only six MPG encoding directions in these data, a logical minimum number for post-processing. This might have made the results vulnerable to small noises and outliers even though the model was simplified to fit small data. Of note, the mean Ds, Df, and fs were in good agreement with the values reported earlier $[9,12,15]$, indicating that the source image data may have had an acceptable quality (Table 2).

This study has several limitations. First, the sample size was not large. Second, the acquired image resolution was low $(64 \times 64)$, which might have caused partial volume effects. Third, the number of MPG encoding directions was the theoretical minimum, six. Vulnerability due to the small data set may have caused some errors throughout the study. Further study with larger sample size and more data may support our results. However, it is important that even the small amount of data did provide useful results with our proposed method, at least with respect to practical application in daily clinics.

Acknowledgments The scientific guarantor of this publication is Takayuki Obata. The authors of this manuscript declare no relationships with any companies, whose products or services may be related to the subject matter of the article. The authors state that this work has not received any funding. No complex statistical methods were necessary for this paper. Institutional review board approval was obtained. Written informed consent was obtained from all subjects (patients) in this study. Methodology: retrospective, observational, performed at one institution.

Open Access This article is distributed under the terms of the Creative Commons Attribution Noncommercial License which permits any noncommercial use, distribution, and reproduction in any medium, provided the original author(s) and the source are credited.

\section{References}

1. Kutzelnigg A, Lucchinetti CF, Stadelmann C, Bruck W, Rauschka H, Bergmann $\mathrm{M}$ et al (2005) Cortical demyelination and diffuse white matter injury in multiple sclerosis. Brain 128:2705-2712, PubMed PMID: 16230320

2. Moll NM, Rietsch AM, Thomas S, Ransohoff AJ, Lee JC, Fox R et al (2011) Multiple sclerosis normal-appearing white matter: pathologyimaging correlations. Ann Neurol 70:764-773, PubMed PMID: 22162059; PubMed Central PMCID: PMC3241216

3. Yoshida M, Hori M, Yokoyama K, Fukunaga I, Suzuki M, Kamagata $\mathrm{K}$ et al (2013) Diffusional kurtosis imaging of normal-appearing white matter in multiple sclerosis: preliminary clinical experience. Jpn J Radiol 31:50-55, PubMed PMID: 23086313

4. Le Bihan D (2007) The 'wet mind': water and functional neuroimaging. Phys Med Biol 52:R57-R90, PubMed PMID: 17374909

5. Rocca MA, Cercignani M, Iannucci G, Comi G, Filippi M (2000) Weekly diffusion-weighted imaging of normal-appearing white matter in MS. Neurology 55:882-884, PubMed PMID: 10994017

6. Cercignani M, Iannucci G, Filippi M (1999) Diffusion-weighted imaging in multiple sclerosis. Ital J Neurol Sci 20:S246-S249, PubMed PMID: 10662958

7. Phuttharak W, Galassi W, Laopaiboon V, Laopaiboon M, Hesselink JR (2007) Abnormal diffusivity of normal appearing brain tissue in multiple sclerosis: a diffusion-weighted MR imaging study. J Med Assoc Thai 90:2689-2694, PubMed PMID: 18386722

8. Tachibana Y, Aida N, Niwa T, Nozawa K, Kusagiri K, Mori K et al (2013) Analysis of multiple B-value diffusion-weighted imaging in pediatric acute encephalopathy. PLoS One 8:e63869, PubMed PMID: 23755112; PubMed Central PMCID: PMC3670889

9. Clark CA, Le Bihan D (2000) Water diffusion compartmentation and anisotropy at high $b$ values in the human brain. Magn Reson Med 44: 852-859, PubMed PMID: 11108621

10. Maier SE, Vajapeyam S, Mamata H, Westin CF, Jolesz FA, Mulkern RV (2004) Biexponential diffusion tensor analysis of human brain diffusion data. Magn Reson Med 51:321-330, PubMed PMID: 14755658

11. Brugieres P, Thomas P, Maraval A, Hosseini H, Combes C, Chafiq A et al (2004) Water diffusion compartmentation at high $b$ values in ischemic human brain. AJNR Am J Neuroradiol 25:692-698, PubMed PMID: 15140706

12. Grinberg F, Farrher E, Kaffanke J, Oros-Peusquens AM, Shah NJ (2011) Non-Gaussian diffusion in human brain tissue at high bfactors as examined by a combined diffusion kurtosis and biexponential diffusion tensor analysis. NeuroImage 57:1087-1102, PubMed PMID: 21596141

13. Sijens PE, Irwan R, Potze JH, Mostert JP, De Keyser J, Oudkerk M (2006) Relationships between brain water content and diffusion tensor imaging parameters (apparent diffusion coefficient and fractional anisotropy) in multiple sclerosis. Eur Radiol 16:898-904, PubMed PMID: 16331463

14. Polman CH, Reingold SC, Banwell B, Clanet M, Cohen JA, Filippi $M$ et al (2011) Diagnostic criteria for multiple sclerosis: 2010 revisions to the McDonald criteria. Ann Neurol 69:292-302, PubMed PMID: 21387374; PubMed Central PMCID: PMC3084507

15. Maier SE, Bogner P, Bajzik G, Mamata H, Mamata Y, Repa I et al (2001) Normal brain and brain tumor: multicomponent apparent diffusion coefficient line scan imaging. Radiology 219:842-849, PubMed PMID: 11376280 